\title{
LA ENSEÑANZA DE LA COMPOSICIÓN ESCRITA: UN "RENACIMIENTO" OLVIDADO DE NUESTRAS AULAS DE INGLÉS
}

\author{
Ángel Sánchez Escobar
}

\section{Introducción}

El propósito principal de este artículo es reflexionar sobre un "renacimiento" comenzado hace tres décadas en Estados Unidos, que no ha llegado a tener repercusión en nuestras aulas. Se trata de un renacimiento en teoría y metodología de la enseñanza de la composición escrita inglesa como primera lengua y su efecto en la enseñanza del inglés como segunda lengua e incluso del español como primera lengua.

El interés en esta mirada retrospectiva surge, por un lado, ante la necesidad de incluir en los nuevos planes de estudio de Filología tanto Inglesa como Española la enseñanza de la composición como asignatura y, por otro lado, ante la necesidad, entre tanta metodología ecléctica, de aclarar ciertos conceptos fundamentales. Las conclusiones que se extraigan de este artículo, aunque centradas en el aprendizaje de la escritura inglesa, son fácilmente transferibles a la adquisición del español como primera lengua.

En Estados Unidos, desde hace años, la enseñanza de la composición escrita forma parte del curriculum de la escuela secundaria y universidad, lo que ha sido la causa de la gran proliferación de bibliografía sobre el tema. En España, sin embargo, se adolece de buenos estudios en este sentido por la ausencia de dicha materia en nuestras aulas. Los estudiantes llegan a la universidad sin saber organizar sus ideas en un texto escrito--aunque se les exija. Parece como si saber escribir surgiera por arte de magia, o por el simple hecho de saber hablar o leer.

En las siguientes páginas, me voy a referir primordialmente a la composición libre, que es la última etapa en la enseñanza de esta destreza. Antes de llegar a ésta, hay un largo camino en la práctica de la escritura controlada y guiada cuya descripción se sale de los límites fijados en estas páginas.

\section{Un renacimiento en la enseñanza de la composición}

Graves se refiere a este renacimiento en "Renaissance and Reform in the Composition Curriculum" (1981, pp. 417-418), estableciendo una primera etapa con la publicación, en 
1963, por Francis Christensen de "A Generative Rhetoric of the Sentence". Lo innovador de este artículo radicaba en que, para Christensen, la composición era un arte que se podía aprender, y no solamente una habilidad innata a unos cuantos. Este artículo abrió nuevos caminos en teoría e investigación.

La siguiente etapa fue la publicación en 1965 por Corbett de Classical Rhetoric for the Modern Student. En este libro, basado en la retórica clásica, Corbett trataba la composición escrita por primera vez en el siglo como una disciplina sistématica y lógica y no como una mezcolanza de opiniones personales.

A partir de aquí, y en años posteriores, comienzan a surgir diversas teorías sobre la enseñanza de la composición, fundamentalmente cuatro--expresiva, epistemológica, retórica y formalista--que se podrían definir, para mayor claridad, con respecto al triángulo de la comunicación:

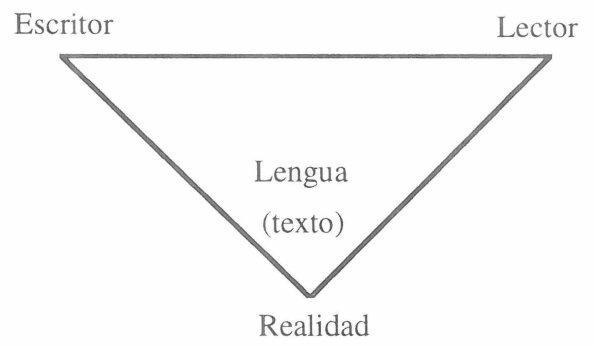

Todas estas teorías tienen que ver con los cuatro elementos del triángulo, pero se centran especialmente en uno de ellos. Así, la teoría expresiva se centra en el escritor; la espistemológica, en el escritor y la realidad, o sea en el proceso mismo de la composición; la retórica, en el lector, y la formalista, en el texto. Se podría decir que las teorías expresiva y epistemológica enfatizan cómo crear ideas, mientras que la retórica y la formalista, cómo expresarlas.

Sin embargo, esto no significa que las primeras no presten atención a las consideraciones de estilo y forma o que las segundas ignoren al escritor, sino que cada una concede interés especial a una fase de la composición e interés secundario a la otra. Veamos estas teorías en detalle:

\section{A) Atención al escritor: el método expresivo}

Este enfoque cubre un amplio espectro, pero la característica común es la programación de las actividades para motivar al estudiante al descubrimiento de sí mismo mediante la expresión de la verdad ("truthtelling"). Ken Macrorie in Telling Writing (1976, pp. 262268) define tres objetivos importantes para la clase de inglés:

(a) elevar el nivel de "truthtelling"; 
(b) animar a los estudiantes a que pierdan su miedo a la clase y al profesor, y que encuentren una auténtica voz en sus escritos;

(c) crear un seminario en el que los estudiantes se ayuden unos a otros en la disciplina del arte de escribir.

Macrorie también ofrece otras actividades para motivar "truthtelling". Algunas de éstas son composición libre, copiar el mejor de los trabajos, dar notas basadas en "truthtelling", comentarios de los otros alumnos antes que el profesor los vea, y la promesa de publicar los mejores trabajos. Macrorie no se refiere a confesiones cuando habla de "truthtelling", sino a poder comunicar hechos y saber objetivizar la experiencia para que un lector pueda vivirla y el escritor revivirla.

En resumen, el programa comienza con composición libre y continúa con una autodisciplina en la búsqueda de la verdad. El estudiante, a través de la composición libre, empieza a descubrir sus propias potencialidades en la lengua. Una vez que ha conseguido confianza en sí mismo, se debe prestar atención a las normas de la composición, tales como la puntuación, el párrafo, las notas al pie de página, etc. Este modelo se diferencia del tradicional en que no está basado en textos de grandes autores, sino en la experiencia del estudiante.

También dentro del mismo método expresivo, Lou Kelly habla en su "Open Class" (1975, pp. 1-13) del auto-descubrimiento del alumno a través de la composición. El seminario que propone se centra en la necesidad de que el escritor se revele a sí mismo, y sepa usar su propio lenguaje, y su propia voz. En este seminario, el alumno tendrá que contestar preguntas de un lector, y tendrá que analizar, limitar, organizar o apoyar lo que escribe. Aquí no hay clases teóricas, ni textos, ni libro de ejercicios. El alumno comparte en la clase sus ideas, opiniones y sentimientos. Los ejercicios intentan establecer un diálogo enriquecedor pidiéndole al estudiante que escriba lo que desee.

El profesor, como un miembro más del grupo, no impondrá sus criterios sino que ofrecerá sus ideas y conocimiento, y hará preguntas que pueden ayudar al alumno a organizar, analizar y razonar sus ideas. También contestará a las preguntas que el alumno no sepa responder. El profesor aquí se presenta como la persona que ayuda a decir lo que ellos quieren decir con claridad y fuerza. Las consideraciones de ortografía, puntuación y uso correcto se posponen en la "open class" para la última etapa cuando el trabajo va a ser leído por alguien. Este programa, como el de Macrorie, intenta que el alumno traiga a la clase la realidad de su vida.

En el método expresivo desarrollado por Macrorie, Kelly, y otros como Stephen Judy en "The Experiential Approach: Inner Worlds to Outer Worlds" (1980) and Murray en "Teach Writing as Process not Product (1976), se tiende a desarrollar un programa que permita al estudiante encontrar sus propios temas, su propia forma, y su propio uso de la lengua. No hay clases sino talleres de escritura. Murray dice que incluso el profesor debe compartir lo que escribe con los alumnos. 


\section{B) Atención a la realidad y al escritor: el método epistemológico}

El enfoque epistemológico, postulado por Kenneth Dowst en "The Epistemic Approach," (1980, pp. 66-78) ve el lenguaje como la representación de la percepción del mundo que cada uno tiene. Dowst afirma que el uso que hacemos del lenguaje no solamente refleja sino que determina lo que sabemos, lo que podemos hacer, y, en cierta manera quiénes somos.

Dowst no ve la composición como un medio de expresión, como hace el método expresivo, ni como un medio de comunicación más o menos persuasivo como hace el método retórico ni como la producción de un texto perfecto, como hace el método formalista, sino como la forma más intencionada del uso del lenguaje, como un medio de conocer y llegar a conocer. El método epistemológico ve la enseñanza de la composición como un medio de comprender el mundo, de descubrimiento y auto-definición; es decir como un medio de conocer y como un medio de llegar a conocer.

Coles en "The Teaching of Writing as Writing" (1975, pp. 30-33) ofrece un curso de composición en términos parecidos a Dowst. Coles dice que no es un curso en metodología aunque tiene que ver con el desarrollo de ciertas destrezas básicas, sino un curso que intensifica la consciencia del estudiante de la relación entre lenguaje y experiencia. Su tema es la composición concebida no como una manera de decir sino como algo que se dice, como una acción, una extensión del estar en un momento en el tiempo.

Para Dowst y Coles, aprender a escribir es un medio de comprender y descubrir el mundo. Cada ejercicio en este tipo de curso lleva como base la pregunta al estudiante de ¿dónde te sitúas tú? Pero ese tú no es equivalente a identidad, sino al tú literario, al tú que se construye al rellenar una página.

El curso delineado por Dowst y Coles no depende de libros de textos sino de una secuencia de ejercicios que se hacen progresivamente complejos en pensamiento y expresión. Se propone al estudiante que aisle un trozo de su experiencia para posteriormente preguntarle qué ha hecho cuando lo ha descrito. Un típico ejercicio de clase sería decirles que describiesen un bloque de pisos, y luego pedirles que expliquen el principio de selección que les llevó a la elección de los detalles. A través de esto, el estudiante se da cuenta del proceso de la composición.

\section{C) Atención al lector: el método rétórico}

Es difícil trazar límites perfectos entre el método retórico y el método formalista, porque la atención al lector nos conduce a una prosa bien escrita y viceversa; pero afinando algo, se podría decir que en el formalista se ve el texto como un fin en sí mismo--aunque tenga que tener en cuenta ciertos principios de legibilidad--mientras que el retórico enfatiza la comunicación de ese texto.

En el método retórico, el texto no está bien o mal en relación a sí mismo sino en relación a un lector o a un propósito predeterminado. Por lo tanto, el mismo tema puede ser 
cambiado para satisfacer diferentes lectores y propósitos. Los proponentes de este método ven la composición como comunicación, y hacen uso de la enseñanza de la retórica clásica.

Larson, en "Teaching Rhetoric in the High School" (1970, pp. 122-129), dice en este sentido que la eficacia de la comunicación se hace realidad cuando el lector comprende y respeta lo que se dice. Larson cree que la tarea del escritor es más clara cuando acude a tres de las cinco partes tradicionales de la retórica: invención (búsqueda de ideas), disposición (la apropiada colocación de estas ideas en el discurso) y elocución (expresión de las ideas en palabras y oraciones). Para Larson, retórica es el arte de adoptar las ideas, estructura y estilo de texto a un determinado lector, ocasión y propósito para el que el discurso se escribe.

Larson diseña un seminario de composición en el que el profesor debe:

-definir un determinado lector al que los estudiantes tienen que dirigir sus escritos, e incluso decirles que compongan el mismo tema con mira a lectores diferentes. Las consideraciones de estilo se verán referidas a este lector.

-pedir al estudiante que seleccione y disponga el material de acuerdo con dicho lector o propósito del tema.

-hacer que analicen escritos actuales y conferencias desde un punto de vista retórico. Este punto puede incluir discusión sobre organización, particular combinación de los datos, estructura de las oraciones, niveles del lenguaje y figuras de la lengua.

-sugerir al estudiante que parodie las diferentes técnicas usadas por los autores que se estudian.

Según Larson, este tipo de práctica haría que el alumno viera la composición no como un ejercicio estéril para hacer en casa o en la clase y compartido sólo con el profesor sino como una manera de transmitir un mensaje a alguien con un propósito. También hace que el estudiante no vea la composición como una tarea para adquirir corrección o tratar de adivinar qué le gusta o no le gusta al profesor, sino como una práctica que requiere la toma de decisiones.

\section{D) Atención al texto: el método formalista}

El método formalista abarca distintas perspectivas: gramática tradicional, gramática transformacional, retórica generativa de la oración, del párrafo y del ensayo; conceptos como legibilidad (readability) y complejidad (complexity); y prácticas en el aula como "sentence combining", y análisis de errores. Para no confundir, el término "retórica" en el método formalista no se refiere a la retórica clasica como propone el método retórico, sino como dice Barry Ulanov (1976, p. 296), al análisis profundo de la lengua, a la psicología de la lengua.

En temas de organización de textos escritos, el método formalista es el que se adecua más a la enseñanza de la composición inglesa en España, y, por motivos de claridad, en relación a ésta lo voy a describir. 
En primer lugar, es necesario admitir que aunque el estudiante tenga un excelente dominio de las estructuras sintácticas del inglés, todavía no dispone de capacidad para componer ensayos, tesis, etc. ¿Por qué? Kaplan en "Inter-cultural Education" (1972, p. 346) afirma que no solamente hay diferencias a nivel de la oración sino diferencias en la concepción misma de la retórica, que varía de cultura en cultura. La retórica del español por su carácter digresivo es diferente de la del inglés, que es linear en su desarrollo.

Esto se ve analizando ensayos de tipo expositivo por escritores profesionales. En éstos el párrafo expositivo siempre comienza con un "topic sentence" o enunciado más general que lleva la idea central de éste, y que luego, por una serie de subdivisiones, procede a desarrollar la idea central en relación propias con otras ideas, para probar algo, o quizás discutir algo. Está claro que la retórica española no se ajusta a esta linearidad. Se desprende que después de tener un dominio de las estructuras sintácticas, el estudiante debe aprender la particular concepción de la composición inglesa, debe aprender los principios de organización.

Para esto, conceptos como la rétorica generativa de la oración, del párrafo y del ensayo estudiados por Irmscher (1979), Paul Rodgers (1976), Christensen (1976) y Becker (1976), podrían ser de mucha utilidad. Estos autores ven la estructura del ensayo como un proceso generativo en el que sus partes, la oración y el párrafo son interdependientes en forma, función y significado. El párrafo y el ensayo, nos dice D'Angelo en "A Generative Rhetoric of the Essay" (1976, p. 202-203), no sólo tiene que tener continuidad y coherencia entre sus partes sino que debe generar un sistema de relaciones semánticas en que las espectativas del lector se cumplan. D'Angelo además observa que los conceptos de coordinación y subordinación, aplicables a nivel sintáctico y semántico, son atribuibles al párrafo y al ensayo. Para él, el ensayo es un tipo de macropárrafo. La primera oración del ensayo es la oración que genera el principio de organización. Las siguientes están relacionadas con ésta por coordinación o subordinación ${ }^{1}$.

En el método formalista, la enseñanza de la oración es importante para entender la organización del párrafo y del ensayo. Pero hay otros conceptos importantes aplicables de igual manera a la enseñanza de la composición. Estos son "relative readability" y "complexity" propuestos por Hirsch y por Williams en The Philosophy of Composition (1970, pp. 75-154) y "Defining Complexity" (1979, pp. 596-608) respectivamente.

Hirsch define "readability" en términos comparativos. Dice que asumiendo que dos textos tengan el mismo significado, el texto más legible será aquel que tome menos tiempo y esfuerzo para entenderlo. Hirsch basa su definición de legibilidad aludiendo a la función semántica de las palabras. El lector cuando lee un texto no recuerda su forma lingüística (formas léxicas o gramaticales) sino que almacena su significado. Para él la legibilidad relativa de un texto se basa más en principios sicológicos que lógicos. La legibilidad se

\footnotetext{
${ }^{1}$ En mi tesis doctoral, A Contrastive Study of the Rhetorical Patterns of English and Spanish Writing, (1984), Nashville, Tennessee, Vanderbilt University, sin publicar, hago un estudio en profundidad de los principios de organización de la escritura expositiva inglesa en contraste con la del español.
} 
logra cuando el texto ofrece economía de atención y consigue canalizar la atención del lector hacia un segmento amplio del texto. Para ello, se debe:

(a) omitir palabras innecesarias;

(b) hacer el párrafo la unidad de la composición;

(c) usar elementos conectores entre oraciones y frases.

Ideas similares expresa Joseph M. Williams al definir "complexity", aunque desde el punto de vista del escritor. Williams explica la dificultad de escribir en un estilo complejo. De acuerdo con Williams, el estudiante debe convertirse en un adulto que sea capaz de comunicarse fácilmente y claramente con un lector que no tenga que luchar para comprender lo que quiere decir.

Williams no está de acuerdo con la idea tradicional de "estilo complejo" que se mide en función del número de subordinadas. Williams define estilo complejo acudiendo al la gramática transformacional, y analiza dos oraciones con identica estructuras superficial:

1. John is eager to please (John está deseoso de agradar)

2. John is easy to please (John es fácil de agradar)

Afirma que la primera es más fácil de procesar porque, como nos indica la estructura profunda, John es el agente de "eager" y "please", lo que significa que hay una correspondencia entre la estructura gramatical y la semántica. En la segunda, sin embargo, la estructura gramatical contradice la semántica. Según Williams las estructuras deben coincidir para poder crear un estilo eficiente al máximo. Esto se debe aplicar no solamente a la oración sino al párrafo y al ensayo completo. Conceptos como "legibilidad relativa" y "estilo complejo" pueden también ayudar al estudiante español a eliminar su tendencia a la digresión, enseñándoles a escribir con claridad y eficientemente.

Dentro del método formalista, también existe abundante práctica de clase diseñada para la adquisición de madurez sintácticas de estudiantes de primera lengua, pero que son aplicables a segunda lengua también, como "sentence combining" y análisis de errores. "Sentence Combining" surgió al intentar invertirse el orden de las transformaciones de la gramática transformacional. La idea fue propuesta for John C. Mellon (1979, pp. 2-3). Él daba al estudiante las transformaciones con ciertas claves y de ahí éste tenía que reconstruir la oración compuesta. Esta idea fue posteriormente desarrollada por Frank O'Hare, William Strong, y el Grupo de la Universidad de Miami--como se le ha venido a llamar.

O'Hare en Sentence Combining: Improving Student Writing without Formal Grammar Instruction (1973) cambió las claves y en lugar de reglas como (T Infinitive), escribió (FOR + TO). Strong en "Sentence Combining back to Basics and Beyond" (1976) configuró un tipo de "open format", y eliminó todas las claves. Un tercer desarrollo por el grupo de Miami (Daiker, Kerek y Moremberg, 1979) fue la aplicación de esta teoría al curso de composición completo, sin claves. (Ver Apéndice)

Strong dice (1976, pp.60-64) que la práctica de "sentence combining" señalizadas o sin señalizar: 
-ayuda a que los estudiantes confíen en sí mismos

-dirige al alumno a hacer ejercicios orales forzándoles así a memorizar cada vez más largas secuencias de lengua.

-hace que se puedan visualizar las oraciones transformadas al requerir que el estudiante las escriba en la pizarra o proyector de transparencias, y discutir en clase otras posibles soluciones.

Strong desarrolla sus ideas en su libro de textos, Sentence Combining: A Composing Book (1973), aplicable a la oración y al párrafo. De las ideas de Strong, Daiker, Kerek y Moremberg crearon un libro de texto, The Writer's Option (1979), también muy valioso porque comienza cada unidad con explicaciones gramaticales. Este libro de texto guía al estudiante desde el manejo de la oración hasta el dominio del ensayo completo. Ofrece una gran variedad de ejercicios y se estudian además problemas de coherencia, puntuación y dicción. (Ver Apéndice)

Indudablemente, la práctica en "sentence combining" ayudaría al estudiante a adquirir destreza en la composición de estructuras de oraciones compuestas, en cierta manera sin acudir a la enseñanza formal de la gramática, y a darse cuenta de su potencial lingüístico. Además, con este tipo de ejercicios el estudiante empieza a desarrollar cierto sentido por lo que "suena mejor", por las opciones estilísticas y por el párrafo. Pero a pesar de esto, el estudiante todavía producirá un gran número de oraciones agramaticales, que se podrían evitar en parte si comprende la naturaleza de los errores. Libros como el de Mina Shaugnessy, Errors and Expectations (1977) aunque aplicado a la primera lengua también podrían dar alguna clave en este sentido.

Estudios en este campo referidos a la adquisición de una segunda lengua como el de Selinker "Interlanguage" (1974) y el de Richards "Error Analysis and Second Language Learning" (1974) son valiosos. Richards (pp. 32-53) identifica las siguientes fuentes de errores:

-por transferencia de la lengua materna a la segunda lengua,

-por generalización de reglas de la primera lengua,

-por cansancio, confusión (errores no sistemáticos),

-por comunicarse sin haber adquirido las formas gramaticales necesarias,

-por metodología empleada por el profesor o el libro de texto.

El análisis de los errores se puede hacer patente a los niveles morfológicos, y sintácticos, y no solamente a nivel de la oración sino del párrafo y del ensayo completo.

\section{Conclusiones}

Aunque cada profesor, a través de su práctica en el aula, debe sacar sus propias conclusiones, se podría admitir que el método expresivo es aplicable a nuestras aulas porque crea un contexto ideal para que el estudiante aporte sus propias experiencias al escribir. Esto les dará algo qué decir y desarrollará en él la confianza en sí mismo que necesita para la 
composición libre. Es decir, motivará al estudiante. Chastain (1988, p. 252) dice respecto al método expresivo: "Teachers seeking to elevate the quality of students' written communication skills can take definite steps toward changing their basic approach to writing as a means of self-expression."

El método epistemológico, por su énfasis en el proceso de la composición más que en ésta misma, tendría aplicabilidad en estudiantes con un elevado nivel de inglés. Este método ayudaría a comprender más la forma de apreciar la realidad del escritor en lengua inglesa y cómo la plasma en un texto. De todas formas, el reconocimiento de este proceso se puede hacer en primera lengua con mejores resultados.

El método retórico puede ayudar al estudiante a adoptar la ideas, estructura y estilo del texto al lector, ocasión y propósito para el que se escribe, en lugar de centrarlo en el profesor. Este método es especialmente importante en la enseñanza del inglés científicotécnico, donde la definición del receptor/ lector es tan importante. Zimmerman y Clark, en Guide to Technical and Scientific Communication (1987, pp. 85-106), en el capítulo entero dedicado a "Audience Analysis", dicen:

In comunicating about your profession, you usually want an audience to know or do something. Important as good organization, proper grammar, and clarity of expression are, they do not guarantee that your audience will understand you. Even the simplest organization and shorterst words make assumptions about those who read or listen to a message. The most dangerous assumptions you can make is to assume that your audience has the same frame reference -knowledge, experience, opinions, and attitudesas you.

Por último, el método formalista, al centrarse en el texto en sí, su organización y capacidad generativa, representa un gran apoyo en nuestras clases de inglés como segunda lengua. A través de éste, los alumnos adquieren madurez sintáctica y se contrarresta la tendencia a la digresión del español. La mayoría de los textos sobre la enseñanza de la escritura utilizan este método en un gran porcentaje, aunque quizás no desarrollen todo su potencial.

Una vez más, quizás sea el eclecticismo la palabra "mágica" que resuene en nuestras mentes tras estas páginas. Se seguirán escribiendo más artículos, algunos, como éste, intentando volver atrás para clarificar y reivindicar la enseñanza de la composición, otros con nuevas investigaciones, otros aplicando lo investigado a cursos prácticos. Pero lo importante, la aplicación a nuestras aulas, sigue brillando por su ausencia en España desde los niveles de enseñanza secundaria hasta los de la facultad. Se necesitan cursos escolares completos dedicados a la enseñanza de la composición inglesa o española. Los tiempos de oscurantismo en los que se pensaba que la escritura surge espontáneamente del habla han pasado ya, ¿o no?. 


\section{BIBLIOGRAFÍA}

BECKER, A.L., (1976) "A Tagmemic Approach to Paragraph Analysis", Rhetoric and Composition, Rochelle Park, N.J., Hayden Book, pp. 154-161.

CHASTAIN, KENNETH, (1988), Developing Second-Language Skills: Theory and Practice, Orlando, Florida, Harcourt Brace Jovanovich.

CHRISTENSEN, FRANCIS, (1976), "A Generative Rhetoric of the Sentence", en Rhetoric and Composition, Rochelle Park, N.J., Hayden Book, pp. 129-138.

CHRISTENSEN, FRANCIS, (1976), "A Generative Rhetoric of the Paragraph", en Rhetoric and Composition, Rochelle Park, N.J., Hyden Book Company, pp. 162-178.

COLES, JR. W.E., (1975), "The Teaching of Writing as Writing" Ideas for English 101, Urbana, Illinois, NCTE, pp. 30-35.

CORBETT, EDWARD, P.J.,(1967), Classical Rhetoric for the Modern Student, $2^{\mathrm{a}}$ edición, Oxford University Press, 1971.

DAIKER, DONALD A.; KEREK, ANDREW; Y MOREMBERG, MAX, (1979), The Writer's Option: College Sentence Combining, N.Y., Harper and Row.

D'ANGELO, FRANK, (1976), "A Generative Rhetoric of the Essay", Rhetoric and Composition, pp. 201-211, Rochelle Park, N.J., Hayden Book.

DOWST, KENNETH, (1980), "The Epistemic Approach: Writing, Knowing, and Learning," en Eight Approaches to Teaching Composition, Illinois, NCTE, pp. 69-73.

GRAVES, RICHARD L., (1981), "Renaissance and Reform in the Composition Curriculum", Phi Delta Kappan, Feb, pp. 417-420.

HIRSCH, E. D., JR, (1970) The Philosophy of Composition, Chicago, The University of Chicago Press, 1970.

IRMSCHER, WILLIAM, F., (1979) Teaching Expository Writing, New York, Holt, Rinehart and Winston.

JACK C., (1974), "Error Analysis and Second Language Learning" en New Frontiers in the Second Language Learning, Boston, Mass., Newbury Publishers, pp. 33-53.

JUDY, STEPHEN, (1980), "The Experiential Approach: Inner Worlds to Outer Worlds," en Eight Approaches to Teaching Composition, Illinois, NCTE, pp. 37-51.

KAPLAN, ROBERT, B., (1972) "Inter-cultural Education" Readings on English a Second Language, Cambridge, Winthrop Publishers, pp. 245-262

KELlY, LOU, (1975), "Toward Competence and Creativity in an Open Class", en Ideas for English 101, Urbana, illinois, NCTE, pp. 2-18.

LARSON, RICHARD, (1970), "Teaching Rhetoric in the High School: Some Proposals," en Teaching High School Composition, New York, Oxford University Press, pp. 121-130.

MACRORIE, KEN, (1976), Telling Writing, New York, Hayden Book.

MELLON, JOHN C., (1979), "Issues in the Theory and Practice of Sentence Combining: A Twenty-year Perspective," en Sentence Combining and the Teaching of Writing, Akron, Ohio, pp. 1-38. 
MURRAY, DONALD M., (1976), "Teach Writing as Process not Product," en Rhetoric and Composition, New York, Hayden Book, pp. 79-82.

O'HARE, FRANK, (1973), Sentence Combining: Improving Student Writing without Formal Grammar Instruction, Urbana, Illinois, NCTE.

RODGERS, PAUL, (1976), "The Stadium of Discourse", Rhetoric and Composition, Rochelle Park, N.J., Hayden Book, pp. 179-182.

SÁNCHEZ ESCOBBAR, ÁNGEL, (1984), A Contrastive Study of the Rhetorical Patterns of English and Spanish Writing, Nashville, Tenneesee, Vanderbilt University, sin publicar.

SELINKER, LARRY, (1974) "Interlanguage", en New Frontiers in Second Language Learning, Boston, Mass., Newbury Publishers, pp. 114-136.

SHAUGNESSY, MINA P., (1977), Errors and Expectations, New York, Oxford University Press.

STRONG, WILliAM, (1973), Sentence Combining: A Composing Book, N.Y., Random House.

ULANOV, BARRY, (1976), "The Relevance of Rhetoric," en Rhetoric and Composition, Rochelle Park, N.J., Hayden Book, pp. 296-302.

WILliAMS, JOSEPH M., (1979), "Defining Complexity", College English, 40, n 6, February, pp. 595-609.

ZIMMERMAN, DONALD E. Y CLARK, DAVID G. (1987), Guide to Technical and Scientific Communication, New York, Random House. 


\section{APÉNDICE}

\section{EJERCICIOS DE "SENTENCE COMBINING" DE O'HARE}

En cada uno de los ejemplos, la forma "A" es el ejercicio dado al estudiante y la "B" la solución aceptable.

\section{THAT Y THE FACT THAT}

A. Peter noticed SOMETHING

There were nine golf balls in the river. (THAT)

B. Peter noticed that there were nine balls in the river.

\section{A. SOMETHING should make you avoid him.}

$\mathrm{He}$ is an absolute nut. (THE FACT THAT)

B. The fact that he is an absolute nut should make you avoid him.

\section{WHICH/THAT, WHO and WHOM}

A. In his letter Ralph enclosed a snapshot.

He had taken a snapshot during his visit with us. (WHICH/THAT)

B. In his letter Ralph enclosed a snapshot which he had taken during his visit with us.

\section{EJERCICIOS DE SENTENCE COMBINING: A COMPOSING BOOK DE STRONG}

\section{Rock Concert}

1. The singer was young.

3. He stepped into the spotlight.

5. His shirt was unbuttoned.

2. The singer was swarthy.

7. Sounds ballooned around him.

4. The spotlight was red.

6. The unbuttoned shirt bared his chest.

9. The sounds were of drums.

8. The sounds were of guitars.

11. The girls were screaming.

10. The sounds were of girls.

The singer, who was young and swarthy, stepped into the red sportlight. His unbuttoned shirt bared his shirt. Sounds of guitar, drums, and screaming girls ballooned around him.

\section{EJERCICIOS DE THE WRITER'S OPTION DE DAIKER-KELEK-MOREMBERG}

Basic Pattern Exercise

Combina cada uno de los grupos de oraciones que siguen y forma una oración que contenga al menos un giro absoluto:

1. When I walked in, Grandpa was sitting at the kitchen table

2. The newspaper was spread before him.

When I walked in, Grandpa was sitting at the kitchen table, the newspaper spread before him.

\section{EJERCICIOS DE CHRISTENSEN}

Prepositional Phrases

Completa la oración como se indica:

1. The old man gazed

2. a/an figure (prep.phrase--descriptive detail of man) (prep.phrase--direction or place)

(modificador) (prep. phrase--descriptive details of "figure" 3.

(prep. phrase--comparison) 